\title{
Physiotherapeutische von heilpraktischer Behandlung trennen?
}

\section{Die Rechtsfrage}

„ Soweit ich weiß, muss ich meine Tätigkeiten als

Physiotherapeutin und Heilpraktikerin räumlich

und zeitlich eindeutig voneinander trennen. Ich

darf also nicht auf derselben Bank und innerhalb

einer Sitzung erst 20 Minuten auf einer Physio-

therapieverordnung behandeln und dann weitere

10 Minuten als sektorale Heilpraktikerin. Richtig? «

Susanne Ehrchen aus Hamburg

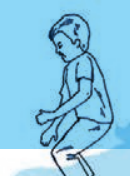

23)

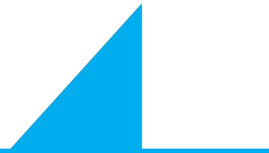

.
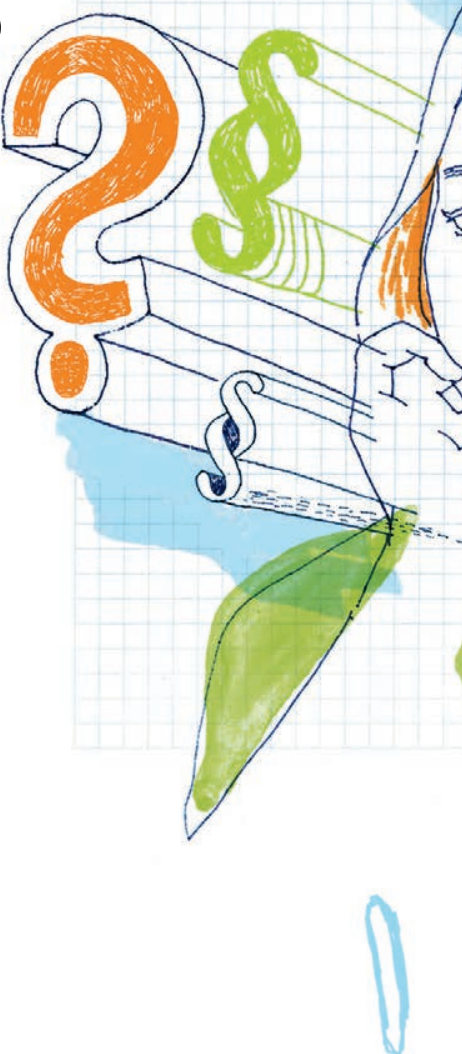

Die Antwort unseres Experten

Gerichtliche Entscheidungen über die räumliche Trennung einer ärztlich verordneten und einer nicht ärztlich verordneten physiotherapeutischen Behandlung sind mir nicht bekannt. Auch aus sonstigen rechtlichen Materialien lässt sich kein Gebot herleiten, das besagt, dass Sie die Leistungen in getrennten Räumen erbringen müssen.

Relevant kann die Frage allenfalls aus kassenzulassungsrechtlicher Sicht sein. Aus den Zulassungsempfehlungen geht hervor, dass eine Praxis in sich abgeschlossen und von anderen Praxen sowie privaten und gewerblichen Bereichen räumlich getrennt sein muss. Diese Regelung dürfte vor allem dem Schutz der Patienten dienen. Wer nur Leistungen an privat versicherte Patienten oder Selbstzahler abgibt, ist hiervon nicht betroffen.

Ein Verbot, physiotherapeutische Behandlungen ohne ärztliche Verordnung in der Praxis abzugeben, kann aus dieser
Regelung allerdings nicht hergeleitet werden. Insbesondere erbringen Physiotherapeuten neben Kassenleistungen auch Selbstzahlerleistungen, ohne dadurch ihre Kassenzulassung zu gefährden. Ob die Selbstzahlerleistung eine Massage ist oder eine Manuelle Therapie, dürfte ohne Belang sein. Für Letztere benötigt man allerdings aus strafrechtlichen Gründen zumindest eine beschränkte Heilpraktikererlaubnis.

Eine räumliche Trennung dürfte daher zumindest für solche Behandlungen, die auch ärztlich verordnet werden können, rechtlich nicht erforderlich sein. Die Notwendigkeit für eine Trennung bei identischen Behandlungen ist nicht im Ansatz erkennbar und verstieße möglicherweise auch gegen die verfassungsmäßig garantierte Berufsfreiheit. Philipp Groteloh

$\Rightarrow$ Wirft auch Ihr Berufsalltag rechtliche Fragen auf? Dann schreiben Sie an Simone.Gritsch@thieme.de.

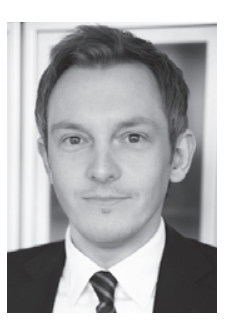

Dr. Philipp Groteloh ist seit 2007 Rechtsanwalt und seit 2012 Fachanwalt für Verwaltungsrecht. 